\title{
Atomistic ensembles of proteins and soft matter complexes from MD simulations and solution scattering data
}

\author{
$\mathrm{J} \mathrm{Hub}^{1}$ \\ ${ }^{1}$ Saarland University, Theoretical Physics, Saarbrücken, Germany \\ jochen.hub@uni-saarland.de
}

Understanding the function of disordered proteins or soft-matter complexes requires understanding of their conformational ensembles. However, experimental data alone is often insufficient for defining all degrees of freedom of such systems, whereas simulations may be biased by poor sampling or force field limitations. We developed a method for coupling atomistic simulations on-the-fly to small- and wide-angle X-ray scattering (SAXS/WAXS) data, based on Jaynes' principle of maximum entropy, with the aim to obtain accurate atomistic ensembles biomolecular and soft-matter systems. As examples, we show that the method is capable of overcoming force field inaccuracies in simulations of an intrinsically disordered protein and of a detergent micelle. In addition, we critically review capabilities and limitations of widely used continuum models in deriving structures of softmatter complexes.

[1] Hub, Curr Opin Struct Biol, 49, 18-26 (2018)

[2] Hermann and Hub, J Chem Theory Comput, 15, 95103-5115 (2019)

[3] Ivanović, Bruetzel, Lipfert, Hub, Angew Chem Int Ed, 57, 5635-5639 (2018)

[4] Ivanović, Hermann, Wójcik, Pérez, Hub, J. Phys. Chem. Lett., 11, 945-951 (2020)

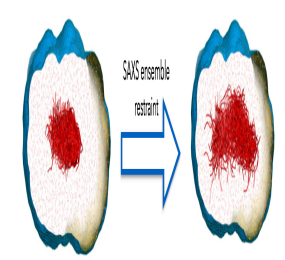

Figure 1

Acta Cryst. (2020). A76, a68 